\title{
The Neural Mechanisms of Tinnitus: A Perspective From Functional Magnetic Resonance Imaging
}

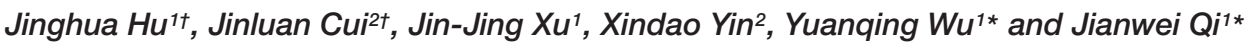 \\ ${ }^{1}$ Department of Otolaryngology, Nanjing First Hospital, Nanjing Medical University, Nanjing, China, ${ }^{2}$ Department \\ of Radiology, Nanjing First Hospital, Nanjing Medical University, Nanjing, China
}

OPEN ACCESS

Edited by:

Zhenchang Wang,

Beijing Friendship Hospital, Capital Medical University, China

Reviewed by:

Zhenyu Xiong,

University of Texas Southwestern

Medical Center, United States

Vijaya Prakash Krishnan Muthaiah,

University at Buffalo, United States

*Correspondence:

Yuanqing WU

cnnxdd@163.com

Jianwei Qi

qjw70818@163.com

${ }^{+}$These authors have contributed equally to this work

Specialty section:

This article was submitted to Auditory Cognitive Neuroscience, a section of the journal

Frontiers in Neuroscience

Received: 25 October 2020

Accepted: 05 January 2021

Published: 11 February 2021

Citation:

Hu J, Cui J, Xu J-J, Yin X, Wu Y and Qi J (2021) The Neural

Mechanisms of Tinnitus: A Perspective From Functional Magnetic Resonance Imaging.

Front. Neurosci. 15:621145. doi: 10.3389/fnins.2021.621145
Tinnitus refers to sound perception in the absence of external sound stimulus. It has become a worldwide problem affecting all age groups especially the elderly. Tinnitus often accompanies hearing loss and some mood disorders like depression and anxiety. The comprehensive adverse effects of tinnitus on people determine the severity of tinnitus. Understanding the mechanisms of tinnitus and related discomfort may be beneficial to the prevention and treatment, and then getting patients out of tinnitus distress. Functional magnetic resonance imaging (fMRI) is a powerful technique for characterizing the intrinsic brain activity and making us better understand the tinnitus neural mechanism. In this article, we review $\mathrm{fMRI}$ studies published in recent years on the neuroimaging mechanisms of tinnitus. The results have revealed various neural network alterations in tinnitus patients, including the auditory system, limbic system, default mode network, attention system, and some other areas involved in memory, emotion, attention, and control. Moreover, changes in functional connectivity and neural activity in these networks are related to the perception, persistence, and severity of tinnitus. In summary, the neural mechanism of tinnitus is a complex regulatory mechanism involving multiple networks. Future research is needed to study these neural networks more accurately to refine the tinnitus models.

Keywords: tinnitus, neural mechanism, MRI, fMRI, functional connectivity

\section{INTRODUCTION}

\section{Tinnitus}

Tinnitus is defined as a phantom auditory perception without external sound stimulation. Tinnitus is common in otolaryngology, with a prevalence rate of $10-15 \%$ in adults (Henry et al., 2020) and about $32.0 \%$ in an elderly population (Chang et al., 2019). Bothersome tinnitus can cause mood disorders like depression, anxiety, and also leads to sleep disorders. In turn, these disorders can further exacerbate tinnitus (Bhatt et al., 2017). There could be a common neurobiological pathway involved in the development of both depression and tinnitus so that tinnitus sufferers may be particularly susceptible to the stress of the phantom sound (Pinto et al., 2014).

A universally agreed classification system for tinnitus has not been realized yet. Tinnitus can be classified by different methods based on clinical and etiological factors such as pulsatile or non-pulsatile (persistent), subjective or objective, conductive or sensorineural paralleling the way hearing loss is classified (Coelho et al., 2020). Pulsatile tinnitus (PT) is less frequent than nonpulsatile tinnitus (NPT) (Weissman and Hirsch, 2000). PT may be subjective or objective, while 
NPT is subjective only (Weissman and Hirsch, 2000). The mechanisms of PT and NPT are different. The cause of PT can be vascular or myogenic, which can be diagnosed by imaging methods like CT and MRI (Pegge et al., 2017), but the cause of NPT is not entirely clear. Moreover, studies have found that patients with PT have abnormal functional connectivity (FC) in specific areas of the brain. Similar to NPT, long-term PT patients experience changes in auditory cortex and limbic system, which may be related to negative emotions like anxiety and depression (Zheng et al., 2019). There is an fMRI study that found a changed baseline brain activity in some limbic, frontal, and occipital areas. However, because of the relatively clear pathogenesis of $\mathrm{PT}$, the changes in the brain were considered as results following the long-term sound stimulation but not the origin of tinnitus (Lv et al., 2016).

Since NPT is more common than PT clinically, when we use the term "tinnitus," it habitually refers to NPT. Early research on tinnitus focused on auditory pathways. It has been assumed for some time that cochlear dysfunction caused by environmental noise overexposure or cochlear trauma is the trigger of tinnitus (Shore and $\mathrm{Wu}, 2019$ ). However, a small number of studies indicated that many patients still experience tinnitus after the cochlear lesions' complete recovery (Pulec, 1995). Although cochlear dysfunction is strongly associated with tinnitus, CNS must play a main contributory role (Bauer et al., 2008). As a result, an increasing number of researchers have investigated the CNS of a tinnitus brain.

There are several hypotheses about the central mechanism of tinnitus generation. It was found that normal auditory stimuli trigger gamma activity at the thalamus level (Metherate and Cruikshank, 1999), which is enhanced in tinnitus patients (Lorenz et al., 2009). So it was supposed that tinnitus may arise from ongoing inhibitory auditory alpha activity and enhanced gamma activity synchronization (Lorenz et al., 2009). In addition, another important hypothesis advocated that tinnitus originates from central neuroplasticity changes (Jinsheng, 2013; Kapolowicz and Thompson, 2020). It is assumed that the imbalance of excitatory and inhibitory inputs to auditory neurons leads to the plasticity change in the central auditory system (CAS). Sometimes this kind of plasticity adjustment goes beyond the CAS, eventually triggering abnormal activation of multiple systems including increased neuron spontaneous discharges and neural synchrony (Kaltenbach, 2011), so that the non-auditory systems associated with tinnitus deserves comprehensive studies too. The current tinnitus studies increasingly focus on the view that tinnitus is a multisystem problem, which involves memory, emotion, attention, and control networks. They together contribute to the generation and development of tinnitus sound perception and related characteristics (Laureano et al., 2014; Pattyn et al., 2016; Maudoux et al., 2017). Jastreboff (1990) proposed a neuropsychological model that demonstrates that whether tinnitus triggers anxiety or depression depends on whether the limbic systems are involved (Jastreboff and Hazell, 1993). Although there are many studies on the neural mechanism of tinnitus over the years, the exact mode of action and its associated pathophysiology remains an enigma.

\section{Functional Magnetic Resonance Imaging}

During the past several years, the rapid development of neuroimaging techniques has contributed greatly in the noninvasive imaging studies of tinnitus. Increasing numbers of studies on tinnitus have focused on cerebral FC. Previous electrophysiological studies using magnetoencephalography (MEG) or electroencephalography (EEG) have shown alterative neural activities and connectivity in the brain of tinnitus (Nathan et al., 2005; Vanneste and De Ridder, 2012). EEG and MEG techniques have a better temporal resolution but poor anatomical resolution, while functional brain imaging techniques have better structural resolution to make better interpretation on the exact location of the source of the signal (e.g., fMRI) (Han et al., 2016).

The fMRI in a narrow sense only refers to blood oxygen level-dependent fMRI (BOLD-fMRI), which uses blood contrast to observe changes in blood oxygenation in the progress of neural activity. Two main research methods are task-based fMRI and rest-state fMRI (rs-fMRI). In studies of tinnitus mechanism and treatment, task-based fMRI design generally utilizes sound stimulus to induce the blood oxygen changes in some related areas (James et al., 2017; Zimmerman B. et al., 2019), while rsfMRI is usually used to analyze the FC between different seeds in regions of interest (ROI). Compared to task-based fMRI, the rs-fMRI technique does not require complex task design and cooperation of patients with the task (Sair et al., 2016), so that it can directly and comprehensively reflects the spontaneous nerve activity and functional connection networks. Since tinnitus is characterized by highly subjective sound hallucinations, and there is no task-based modulation of the tinnitus signal, rs-fMRI has been proven to be a powerful technique for characterizing the intrinsic brain activity in patients with tinnitus (Husain and Schmidt, 2014; Zhou et al., 2019). The amplitude of low frequency fluctuations (ALFF) and regional homogeneity (ReHo) are methods commonly used in rs-fMRI data analysis to reflect different aspects of regional neural activity, while FC density analysis, seed-based FC analysis, independent component analysis (ICA), and graph analysis are used to analyze the FC of different brain regions ( $\mathrm{Lv}$ et al., 2018).

In a broader sense, fMRI also includes diffusion weighted imaging (DWI), perfusion weighted imaging (PWI), susceptibility weighted imaging (SWI), and magnetic resonance spectroscopy (MRS). Diffusion tensor imaging (DTI) is a special form of DWI. It can evaluate the anisotropy of white matter (WM), so that WM fiber bundles can be observed and tracked effectively. DTI was widely used in some neurological disorders, such as Alzheimer's disease (Bigham et al., 2020) and schizophrenia (Ochi et al., 2020). Chen Q. et al. (2020) conducted a tinnitus study by using DTI. The results showed that tinnitus is related to changes in WM integrity in some brain regions including corpus callosum and cingulum. ASL is one kind of PWI technique to quantify cerebral blood flow (CBF) using the labeling arterial blood as an endogenous tracer (Ferré et al., 2013). One of the latest ASL study of tinnitus found decreased $\mathrm{CBF}$ in the auditory and prefrontal cortex. Interestingly, the degree of headache of tinnitus patients is related to the $\mathrm{CBF}$ decrease (Chen Y. C. et al., 2020). SWI shows intrinsic differences 
in magnetic sensitivity between tissues, such as bleeding and abnormal deposition of iron ions, thus reflecting pathological changes (Guo et al., 2020). At present, it is rarely used in the study of subjective tinnitus. In view of an ASL study on CBF changes in a tinnitus patient, it may be valuable for tinnitus research in the future. MRS is an emerging MRI technology. Taking ${ }^{1} \mathrm{H}$ or ${ }^{31} \mathrm{P}$ as the object of spectrum examination, the concentration of metabolite markers can be calculated, such as $\mathrm{N}$-acetyl aspartate (NAA), lactic acid (Lac), choline (Cho), and so on (He et al., 2020). It is usually used in the differential diagnosis of tumors (Hekmatnia et al., 2019) and neurological disease study, such as epilepsy (Abedi-Firouzjah et al., 2020), Alzheimer's disease, and cognitive impairment (Chandra et al., 2019). Studies have explored the connection between chronic tinnitus and mild cognitive impairment. Maybe the MRS technique will be of help in the further related study. Combining two or more fMRI tools can help with a comprehensive analysis of neuropathological changes from multiple perspectives (Yeo et al., 2018). That is called multimodal fMRI analysis technique. A number of researches have combined functional and structural measurements on tinnitus studies (Luan et al., 2019). This technique has a great application foreground for its comprehensiveness.

Thanks to the rapid development of fMRI technology, we have found a range of abnormal changes in both central auditory system (CAS) and some non-auditory system of people with tinnitus (Kim et al., 2012; Maudoux et al., 2017; Minami et al., 2018). In addition, the $\mathrm{DMN}$, the attention system, and some other areas responsible for memory, emotion, attention, and control are involved in tinnitus, as well as the distress associated with these persistent phantom (more on that below). To better understand the neural network of tinnitus, this review provides a critical review of fMRI studies published in recent years on the neuroimaging mechanisms of tinnitus. Figure $\mathbf{1}$ gives an overview of the tinnitus-related brain areas based on fMRI.

\section{Central Auditory System}

Important structures in the auditory pathway of tinnitus patients, such as the cochlear nucleus, lateral colliculus, inferior colliculus nucleus, medial geniculate body, and cerebral auditory cortex, are likely to play a role in the occurrence of the phantom sound of tinnitus (Kris et al., 2014), suggesting that the initial tinnitus signal is produced by hyperactivity in the central auditory pathway (Rauschecker et al., 2010). Structural and functional anomalies of the CAS have been found in tinnitus (Cai et al., 2019). Studies have found gray matter and white matter alert in the auditory cortex of tinnitus patients (Boyen et al., 2013; Schmidt et al., 2018). A recent fMRI study found decreased ReHo in the primary auditory cortex and increased ReHo in some secondary auditory regions like supramarginal and angular gyri (Anthony et al., 2019). Emmert et al. combined ASL, taskbased, and rest-state fMRI to study how tinnitus patients regulate their own brain activity by continuous and intermittent feedback. It was found that secondary auditory areas might be more susceptible to intermittent feedback modulation (Emmert et al., 2017). This may be related to a compensation mechanism in these secondary auditory areas of tinnitus.

However, a contradicting result was obtained that shows that there are no significant differences in the auditory cortical FC between chronic subjective tinnitus and the control group after correcting for multiple statistical comparisons (Davies et al., 2014). This result indicated that auditory network connectivity was not modified by the experience of tinnitus. They also found altered FC in brain regions related to attention and emotional

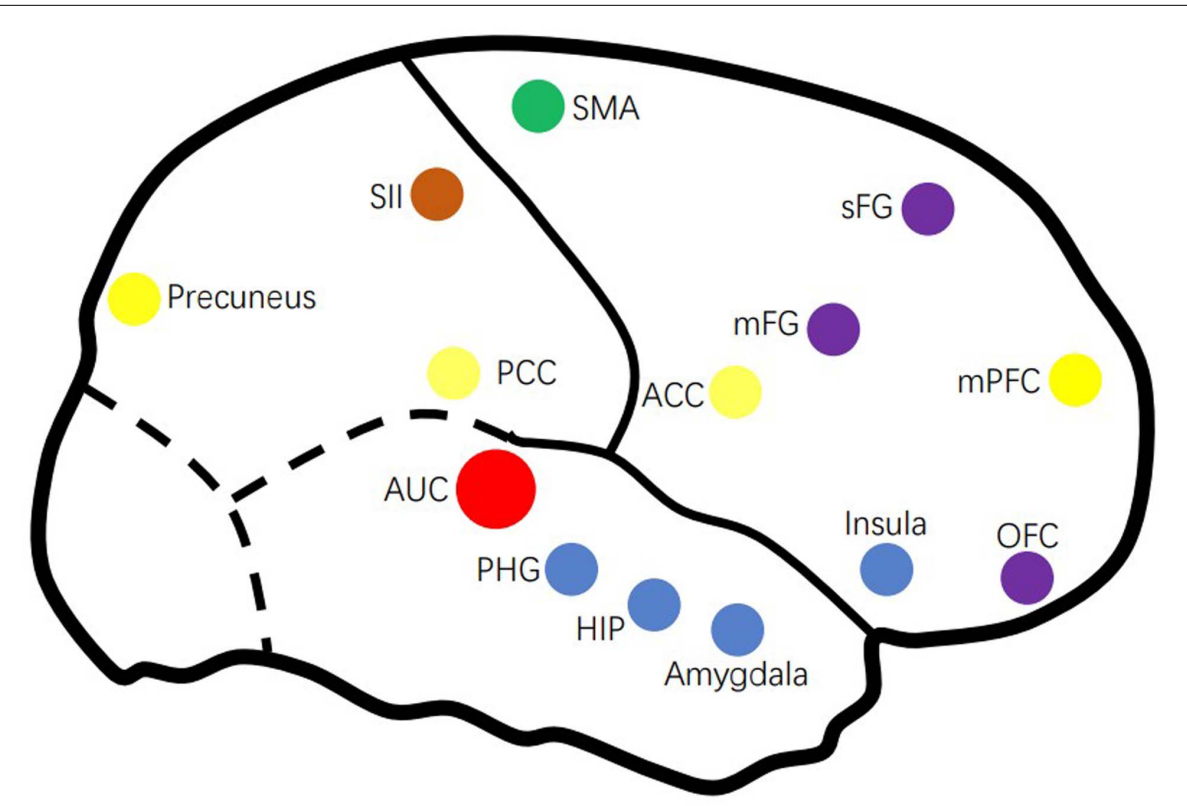

FIGURE 1 | Overview of the tinnitus-related brain areas based on fMRI. AUC, auditory cortex; ACC, anterior cingulate cortex; HIP, hippocampus; mFG, medial frontal cortex; mPFC, medial prefrontal cortex; OFC, orbitofrontal cortex; PCC, posterior cingulate cortex; PHG, parahippocampus; sFG, superior frontal gyrus. 
processing only in bothersome tinnitus. The different result may be caused by many factors such as the tinnitus laterality, the hearing threshold, the severity of the distress, and annoyance. So more studies need to be conducted to compare subgroups with different levels of the abovementioned tinnitus-related features in order to confirm the mechanism of CAS in tinnitus.

Tinnitus is often accompanied by hearing loss but not vice versa (Ovidiu et al., 2006). Some studies attempted to dissociate the effect of tinnitus from hearing loss. It was found that hearing loss causes greater gray and white matter alterations than tinnitus (Husain et al., 2011a), especially in the primary auditory cortex and limbic area of the frontal lobe (Boyen et al., 2013). Hearing loss patients with or without tinnitus have different neural activities and functional changes in the CNS (Kris et al., 2014). Increased connectivity was found between the limbic and auditory regions of hearing loss with tinnitus (Schmidt et al., 2013). To tinnitus patients, hearing loss seems to drive the communication between these two areas and enhance memory-related activity of the hippocampus (Vanneste and De Ridder, 2016). The association of tinnitus and hearing loss makes patients suffer more severe discomfort (Savastano, 2008). Therefore, in the study of the tinnitus neural network, hearing loss should be taken into consideration as an especially important factor.

\section{Limbic System}

The limbic system is termed as the "feeling and reacting brain," which can respond to emotional stimulation. It is also implicated in memory, especially the emotional-related part (Rajmohan and Mohandas, 2007). The composition of the limbic system is controversial, but it includes the parahippocampus, amygdala, hippocampus, anterior cingulate cortex (ACC), and partial basal ganglia (Rajmohan and Mohandas, 2007).

Jastreboff (1990) regarded the limbic system as the main source of psychological responses related to tinnitus for its important role in behavior and emotional expression (Jastreboff and Hazell, 1993). The indispensable role of the limbic system in tinnitus is well established. The initial tinnitus signal may generate from parts of the auditory system, which can be blocked by the limbic system. However, in patients with chronic tinnitus, limbic system damage and resulting inefficiency of auditory-limbic interactions lead to the failure of this inhibition (Rauschecker et al., 2010; Leaver et al., 2016). There were also researchers suggesting that abnormal activation of the limbic structures may occur at an early stage of tinnitus and maintaining this condition throughout the tinnitus period (Kapolowicz and Thompson, 2020). The exact relationship between the limbic system and auditory system has become a hot topic in recent years. Numerous brain imaging studies and conceptual models of tinnitus has demonstrated increased functional and anatomical connectivity between the auditory and limbic regions (Maudoux et al., 2012; Seydell-Greenwald et al., 2014; Chen et al., 2017b).

It is thought that sustained increase in the activity of the parahippocampus leads to adaptive dysfunction, which inhibits adaptation to tinnitus and mis-transmits auditory memory stored in the hippocampus (Sven et al., 2011). The hippocampus and amygdala could also have a role in the persistence of tinnitus by updating auditory memory for tinnitus (Elham and Ghassem, 2018). Gunbey et al. (2017) conducted a DTI study finding a negative relation between the hippocampus-amygdala fractional anisotropy values and tinnitus severity. A recent study applied cyclicity analysis to fMRI data, revealing that the pairs of brain regions connected to the amygdala contributed most to the differences between tinnitus and controls (Zimmerman B. J. et al., 2019). Another fMRI study examined how amygdala acted on emotional sound processing in tinnitus subjects. It suggested enhanced amygdala activation in bothersome tinnitus. To those who successfully adapted to the emotionally negative sound, the emotional response of the amygdala decreased (Davies et al., 2017). These evidences reveal that some of tinnitus brain generators share common resources with brain memory and emotion generators, which may contribute to the persistence of the phantom bothersome sound.

In patients with tinnitus, spontaneous nerve activity and FC are enhanced in the insula (Carpenter-Thompson et al., 2014). The increased response in the insula, mainly the anterior part, may reflect adaption to the tinnitus perception by focusing attention on non-auditory events (van der Loo et al., 2011; Harold et al., 2012). Furthermore, the insula is one of the key nodes in the common brain circuit of both chronic tinnitus and pain (Dirk et al., 2011; Rauschecker et al., 2015). Areas including insula, ventromedial prefrontal cortex (vmPFC), and nucleus accumbens (NAc) act as a central gating system for sensory perception, which determines the sentimental value of sensory stimuli and modulates information transmission in the brain (Rauschecker et al., 2015). Dirk et al. (2011) thought this gating system is a non-specific distress network through the involvement of learning mechanisms and memory mechanisms, and then proposed a working model for the origin of phantom pain and phantom sound. Tinnitus and chronic pain occur when this system is compromised (Chen et al., 2017a). It suggested that tinnitus and neuropathic pain share an overlapping brain network with common activation and connectivity patterns and are differentiated by a specific mechanism (Vanneste et al., 2019). Furthermore, NAc, vmPFC, and subgenual ACC (sgACC) form a frontostriatal top-down gating system, which related to top-down noise cancelation and perceptual learning (Hullfish et al., 2019). A study (Xu et al., 2019) that combined MRI with granger causality analysis (GCA) found directional connectivity were enhanced between the NAc and $\mathrm{PFC}$ in the chronic tinnitus group compared to the control group, and this enhancement was positively correlated with tinnitus duration and distress. It is confirmed that the frontostriatal gating control system is important in tinnitus and pain perception. These hypotheses can help us better understand the mechanism of the distress associated with tinnitus and may extend to other similar phantoms.

\section{Default Mode Network (DMN)}

The DMN is a brain system in which the activity increases in the resting state and decreases in the task state (Shulman et al., 1997). $\mathrm{DMN}$ is generally considered to be composed of the posterior cingulate cortex (PCC)/precuneus, ACC, medial prefrontal cortex(mPFC), and angular gyrus (Mantini et al., 2007). 
Some studies proposed that the generation of tinnitus may be associated with the abnormalities in the DMN. Lanting et al. (2016) conducted an fMRI study combined with independent component analysis (ICA). The data of the two kinds of state (fixed-state and resting state) were obtained for both tinnitus and control group. They found in the tinnitus patients that the DMN was less extensive and showed significantly less connectivity in both states. At the same time, the activity of DMN was not stronger during the resting-state than during the fixed-state. They attribute this pattern to the unremitting engaging effect of the tinnitus perception.

Chronic tinnitus patients show significantly enhanced FC between the ACC and left precuneus, which was correlated with the tinnitus duration (Chen et al., 2018b). In addition, enhanced FC between the PCC and mPFC was positively correlated with the tinnitus distress (Chen et al., 2018a,c). Among them, lack of cognitive control function caused by alterations in PFC may play a pivotal role in tinnitus generation and persistence (SeydellGreenwald et al., 2012; Rodrigo et al., 2018). This result is similar to a previous study of Schmidt et al., which examined two sources of variability in the subgroups: tinnitus severity and duration. They observed decreased correlations between DMN and the precuneus, which were consistent across individuals with longterm tinnitus. More bothersome tinnitus demonstrated stronger decrease. So the connectivity of the precuneus could serve as a marker for long duration tinnitus and index tinnitus severity (Schmidt et al., 2017). They hypothesize that the enhancement of DMN-precuneus connectivity could reduce the severity of tinnitus. The role of DMN in tinnitus was verified from another aspect that the neurofeedback training of the PCC can relieve tinnitus-related distress (Sven et al., 2018). Moreover, there are studies that revealed that DMN was related to the severity of mood symptoms and cognitive dysfunction in some mental illnesses like Alzheimer's disease, schizophrenia, and infantile autism (Binnewijzend et al., 2012; Olivito et al., 2017; Lee et al., 2019). Perhaps DMN plays a similar role in tinnitus, so a neural model of these similar mental illness can be a reference.

\section{Attention and Control System}

There is a lot of evidence indicating that some cortical area of the brain, particularly those networks responsible for attention, focus, and cognitive control also have abnormal activity and FC in tinnitus (Harold et al., 2012).

Attentional network can be divided into the dorsal attention network (DAN) and ventral attention network (VAN). The DAN comprises the intraparietal sulcus (IPS) and the frontal eye fields (FEF), while the the VAN comprises the temporoparietal junction (TPJ) and the ventral frontal cortex (VFC) (Vossel et al., 2014). Many fMRI studies have focused on these brain regions. Husain et al. (2011b) found a decreased activation in the DAN areas, which may be involved in auditory attention and short-term memory network, and a pivotal difference between tinnitus and hearing loss in neural bases. Schmidt et al. (2013, 2017) found an increased FC in tinnitus patients between the DAN and parahippocampus as well as the precuneus, and that was associated with tinnitus severity. Besides, it was found in bothersome tinnitus that the FC for primary visual cortex involved significant negative correlations in CAS, VAN, and some executive control network components. It indicated a dissociation activity between visual, auditory, attention, and control networks in tinnitus. That might reflect a mechanism of adaptation to chronic bothersome tinnitus that reduces the salience of phantom noises and directing attention to nonauditory events (Burton et al., 2012). These studies suggested that improving the function of attention system and mitigating the connectivity between limbic regions, attention, and auditory system could be an effective therapy to reduce tinnitus and related distress.

The cognitive control network (CCN), which directs attentional focus may have a basic role in tinnitus maintenance (Trevis et al., 2016). Decreased activation of the CCN (right middle frontal gyrus is the core node), decreased connectivity between the CCN and salience network (SN), and increased connectivity between the $\mathrm{CCN}$ and autobiographical memory networks (AMN) were found in tinnitus (Trevis et al., 2017). The dysfunction and ineffective connection of $\mathrm{CCN}$ leading to failure of switching attention away from the noisy environment to a task that requires cognition, contributes to persistent awareness of the phantom sound (Trevis et al., 2017).

\section{CONCLUSION}

Combined with prior fMRI research literatures on tinnitus neural network, it is believed that both auditory and nonauditory systems play an important role in tinnitus. The damaged structure and function of the auditory system leads to the origin of tinnitus, but the non-auditory system is indispensable in the continuous perception of tinnitus and is related to some tinnitus characteristics such as duration, severity, loudness, and tinnitus-related distress. Future research should confirm whether the neural network changes found in non-auditory areas of the brain of tinnitus patients are a cause of tinnitus or the result of tinnitus signals being continuously transmitted in these networks. fMRI is certainly a useful tool for exploring neural networks of the tinnitus and similar mental disorders. Research combined with multiple MRI tools would be more helpful. A relatively explicit mechanism can contribute to therapeutical intervention of tinnitus.

\section{AUTHOR CONTRIBUTIONS}

$\mathrm{JH}$ and JC checked the references and wrote the manuscript. J-JX and XY helped review and revise the manuscript. YW and JQ contributed to the discussion and manuscript revision. All authors contributed to the article and approved the submitted version.

\section{FUNDING}

This work was supported by the Youth Medical Talents of Jiangsu Province (No. QNRC2016062) and the 333 High-level Talents Training Project of Jiangsu Province (No. BRA2019122). 


\section{REFERENCES}

Abedi-Firouzjah, R., Rostamzadeh, A., Banaei, A., Shafiee, M., Moghaddam, Z. M., and Vafapour, H. (2020). Exploring changes in thalamus metabolites as diagnostic biomarkers in idiopathic generalised epilepsy patients using magnetic resonance spectroscopy. Malays. J. Med. Sci. MJMS 27, 78-86. doi: 10.21315/mjms2020.27.1.8

Anthony, G., Jeremy, D., Nicolas, M. D. C., Jean-Luc, P., Emmanuelle, L. B., and Frédéric, V. (2019). Alterations in regional homogeneity in patients with unilateral chronic tinnitus. Trends Hear. 23, 1-12.

Bauer, C. A., Turner, J. G., Caspary, D. M., Myers, K. S., and Brozoski, T. J. (2008). Tinnitus and inferior colliculus activity in chinchillas related to three distinct patterns of cochlear trauma. J. Neurosci. Res. 86, 2564-2578. doi: 10.1002/jnr. 21699

Bhatt, J. M., Bhattacharyya, N., and Lin, H. W. (2017). Relationships between tinnitus and the prevalence of anxiety and depression. Laryngoscope 127, 466-469. doi: 10.1002/lary.26107

Bigham, B., Zamanpour, S. A., Zemorshidi, F., Boroumand, F., Zare, H., and Initiative, A. S. D. N. (2020). Identification of superficial white matter abnormalities in alzheimer's disease and mild cognitive impairment using diffusion tensor imaging. J. Alzheimer's Dis. Rep. 4, 49-59. doi: 10.3233/adr190149

Binnewijzend, M. A., Schoonheim, M. M., Sanz-Arigita, E., Wink, A. M., van der Flier, W. M., Tolboom, N., et al. (2012). Resting-state fMRI changes in Alzheimer's disease and mild cognitive impairment. Neurobiol. Aging 33, 2018-2028.

Boyen, K., Langers, D. R., De Kleine, E., and Van Dijk, P. (2013). Gray matter in the brain: differences associated with tinnitus and hearing loss. Hear. Res. 295, 67-78. doi: 10.1016/j.heares.2012.02.010

Burton, H., Wineland, A., Bhattacharya, M., Nicklaus, J., Garcia, K. S., and Piccirillo, J. F. (2012). Altered networks in bothersome tinnitus: a functional connectivity study. BMC Neurosci. 13:3.

Cai, W. W., Li, Z. C., Yang, Q. T., and Zhang, T. (2019). Abnormal spontaneous neural activity of the central auditory system changes the functional connectivity in the tinnitus brain: a resting-state functional MRI study. Front. Neurosci. 13:1314. doi: 10.3389/fnins.2019.01314

Carpenter-Thompson, J. R., Akrofi, K., Schmidt, S. A., Dolcos, F., and Husain, F. T. (2014). Alterations of the emotional processing system may underlie preserved rapid reaction time in tinnitus. Brain Res. 1567, 28-41. doi: 10.1016/j.brainres. 2014.04.024

Chandra, A., Dervenoulas, G., Politis, M., and Initiative, A. S. D. N. (2019). Magnetic resonance imaging in Alzheimer's disease and mild cognitive impairment. J. Neurol. 266, 1293-1302.

Chang, N. C., Dai, C. Y., Lin, W. Y., Yang, H. L., Wang, H. M., Chien, C. Y., et al. (2019). Prevalence of persistent tinnitus and dizziness in an elderly population in Southern Taiwan. J. Int. Adv. Otol. 15, 99-105. doi: 10.5152/iao.2019.6257

Chen, Q., Wang, Z., Lv, H., Zhao, P., Yang, Z., Gong, S., et al. (2020). Reorganization of brain white matter in persistent idiopathic tinnitus patients without hearing loss: evidence from baseline data. Front. Neurosci. 14:591. doi: 10.3389/fnins. 2020.00591

Chen, Y. C., Chen, H., Bo, F., Xu, J. J., Deng, Y., Lv, H., et al. (2018a). Tinnitus distress is associated with enhanced resting-state functional connectivity within the default mode network. Neuropsychiatr. Dis. Treat. 14, 1919-1927. doi: 10.2147/ndt.s164619

Chen, Y. C., Hu, J. H., Cui, J. L., Shang, S. A., Yong, W., Xu, J. J., et al. (2020). Headache is associated with aberrant cerebral blood flow in chronic tinnitus revealed by perfusion functional MRI. Res. Square [Epub ahead of print].

Chen, Y. C., Liu, S., Lv, H., Bo, F., Feng, Y., Chen, H., et al. (2018b). Abnormal resting-state functional connectivity of the anterior cingulate cortex in unilateral chronic tinnitus patients. Front. Neurosci. 12:9. doi: 10.3389/fnins. 2018.00009

Chen, Y. C., Wang, F., Wang, J., Bo, F., Xia, W., Gu, J. P., et al. (2017a). Restingstate brain abnormalities in chronic subjective tinnitus: a meta-analysis. Front. Hum. Neurosci. 11:22. doi: 10.3389/fnhum.2017.00022

Chen, Y. C., Xia, W., Chen, H., Feng, Y., Xu, J. J., Gu, J. P., et al. (2017b). Tinnitus distress is linked to enhanced resting-state functional connectivity from the limbic system to the auditory cortex. Hum. Brain Mapp. 38, 2384-2397. doi: 10.1002/hbm. 23525
Chen, Y. C., Zhang, H., Kong, Y., Lv, H., Cai, Y., Chen, H., et al. (2018c). Alterations of the default mode network and cognitive impairment in patients with unilateral chronic tinnitus. Quant. Imaging Med. Surg. 8, 1020-1029. doi: 10.21037/qims.2018.11.04

Coelho, C. B., Santos, R., Campara, K. F., and Tyler, R. (2020). Classification of tinnitus: multiple causes with the same name. Otolaryngol. Clin. North Am. 53, 515-529.

Davies, J., Gander, P. E., Andrews, M., and Hall, D. A. (2014). Auditory network connectivity in tinnitus patients: a resting-state fMRI study. Int. J. Audiol. 53, 192-198. doi: 10.3109/14992027.2013.846482

Davies, J. E., Gander, P. E., and Hall, D. A. (2017). Does chronic tinnitus alter the emotional response function of the amygdala?: a sound-evoked fMRI study. Front. Aging Neurosci. 9:31. doi: 10.3389/fnagi.2017.00031

Dirk, D. R., Belen, E. A., Ranulfo, R., and Berthold, L. (2011). Phantom percepts: tinnitus and pain as persisting aversive memory networks. Proc. Natl. Acad. Sci. U.S.A. 108, 8075-8080. doi: 10.1073/pnas.1018466108

Elham, T., and Ghassem, M. (2018). A different view on the link between tinnitus and cognition; is there a reciprocal link? Int. J. Neurosci. 128, 1188-1198. doi: 10.1080/00207454.2018.1503185

Emmert, K., Kopel, R., Koush, Y., Maire, R., Senn, P., Van De Ville, D., et al. (2017). Continuous vs. intermittent neurofeedback to regulate auditory cortex activity of tinnitus patients using real-time fMRI - A pilot study. NeuroImage Clin. 14, 97-104. doi: 10.1016/j.nicl.2016.12.023

Ferré, J. C., Bannier, E., Raoult, H., Mineur, G., Carsin-Nicol, B., and Gauvrit, J. Y. (2013). Arterial spin labeling (ASL) perfusion: techniques and clinical use. Diagn. Intervent. Imaging 94, 1211-1223. doi: 10.1016/j.diii.2013.06.010

Gunbey, H. P., Gunbey, E., Aslan, K., Bulut, T., Unal, A., and Incesu, L. (2017). Limbic-auditory interactions of tinnitus: an evaluation using diffusion tensor imaging. Clin. Neuroradiol. 27, 221-230. doi: 10.1007/s00062-015-0473-0

Guo, X., Ma, L., Li, H., Qi, X., Wei, Y., Duan, Z., et al. (2020). Brainstem iron overload and injury in a rat model of brainstem hemorrhage. J. Stroke Cerebrovas. Dis. 29:104956. doi: 10.1016/j.jstrokecerebrovasdis.2020.10 4956

Han, Y., Lei, D., Min, Z., Vadim, Z., Raquel, P., and Jerzy, B. (2016). Reconstructing large-scale brain resting-state networks from high-resolution EEG: spatial and temporal comparisons with fMRI. Brain Connect. 6, 122-135. doi: 10.1089/ brain.2014.0336

Harold, B., Andre, W., Mousumi, B., Joyce, N., Keith, S. G., and Jay, F. P. (2012). Altered networks in bothersome tinnitus: a functional connectivity study. BMC Neurosci. 13:3. doi: 10.1186/1471-2202-13-3

He, Y. N., Meng, Y., Gao, N.-N., Zhang, B.-J., Li, H., and Ji, S.-S. (2020). Effect of injecting mouse nerve growth factor in different ways on motor development, cerebral hemodynamics and biochemical metabolism in children with cerebral palsy. Chinese Acupuncture Moxibustion 40, 839-844.

Hekmatnia, A., Sabouri, M., Ghazavi, A. H., Far, P. S., Hekmatnia, F., Sofi, G. J., et al. (2019). Diagnostic value of magnetic resonance spectroscopy (MRS) for detection of brain tumors in patients. Med. Sci. 23, 939-945.

Henry, J. A., Reavis, K. M., Griest, S. E., Thielman, E. J., Theodoroff, S. M., Grush, L. D., et al. (2020). Tinnitus: an epidemiologic perspective. Otolaryngol. Clin. North Am. 53, 481-499.

Hullfish, J., Abenes, I., Yoo, H. B., De Ridder, D., and Vanneste, S. (2019). Frontostriatal network dysfunction as a domain-general mechanism underlying phantom perception. Hum. Brain Mapp. 40, 2241-2251. doi: 10. 1002/hbm. 24521

Husain, F. T., Medina, R. E., Davis, C. W., Szymko-Bennett, Y., Simonyan, K., Pajor, N. M., et al. (2011a). Neuroanatomical changes due to hearing loss and chronic tinnitus: a combined VBM and DTI study. Brain Res. 1369, 74-88. doi: 10.1016/j.brainres.2010.10.095

Husain, F. T., Pajor, N. M., Smith, J. F., Kim, H. J., Rudy, S., Zalewski, C., et al. (2011b). Discrimination task reveals differences in neural bases of tinnitus and hearing impairment. PLoS One 6:e26639. doi: 10.1371/journal.pone.0026639

Husain, F. T., and Schmidt, S. A. (2014). Using resting state functional connectivity to unravel networks of tinnitus. Hear. Res. 307, 153-162. doi: 10.1016/j.heares. 2013.07.010

James, G. A., Thostenson, J. D., Brown, G., Carter, G., Hayes, H., Tripathi, S. P., et al. (2017). Neural activity during attentional conflict predicts reduction in tinnitus perception following rTMS. Brain Stimul. 10, 934-943. doi: 10.1016/j. brs.2017.05.009 
Jastreboff, P. J. (1990). Phantom auditory perception (tinnitus): mechanisms of generation and perception. Neurosci. Res. 8, 221-254. doi: 10.1016/01680102(90)90031-9

Jastreboff, P. J., and Hazell, J. W. (1993). A neurophysiological approach to tinnitus: clinical implications. Br. J. Audiol. 27, 7-17. doi: 10.3109/03005369309077884

Jinsheng, Z. (2013). Auditory cortex stimulation to suppress tinnitus: mechanisms and strategies. Hear. Res. 295, 38-57. doi: 10.1016/j.heares.2012.05.007

Kaltenbach, J. A. (2011). Tinnitus: models and mechanisms. Hear. Res. 276, 52-60. doi: $10.1016 /$ j.heares.2010.12.003

Kapolowicz, M. R., and Thompson, L. T. (2020). Plasticity in limbic regions at early time points in experimental models of tinnitus. Front. Syst. Neurosci. 13:88. doi: $10.3389 /$ fnsys.2019.00088

Kim, J. Y., Kim, Y. H., Lee, S. H., Seo, J. H., Song, H. J., Cho, J. H., et al. (2012). Alteration of functional connectivity in tinnitus brain revealed by resting-state fMRI?: a pilot study. Int. J. Audiol. 51, 413-417. doi: 10.3109/14992027.2011. 652677

Kris, B., Emile, D. K., Pim, V. D., and Langers, D. R. M. (2014). Tinnitus-related dissociation between cortical and subcortical neural activity in humans with mild to moderate sensorineural hearing loss. Hear. Res. 312, 48-59. doi: 10. 1016/j.heares.2014.03.001

Lanting, C., Wozaniak, A., Van Dijk, P., and Langers, D. R. M. (2016). Tinnitusand task-related differences in resting-state networks. Adv. Exp. Med. Biol. 894, 175-187. doi: 10.1007/978-3-319-25474-6_19

Laureano, M. R., Onishi, E. T., Bressan, R. A., Castiglioni, M. L. V., Batista, I. R., Reis, M. A., et al. (2014). Memory networks in tinnitus: a functional brain image study. PLoS One 9:e87839. doi: 10.1371/journal.pone.0087839

Leaver, A. M., Seydell-Greenwald, A., and Rauschecker, J. P. (2016). Auditorylimbic interactions in chronic tinnitus: challenges for neuroimaging research. Hear. Res. 334, 49-57. doi: 10.1016/j.heares.2015.08.005

Lee, H., Lee, D. K., Park, K., Kim, C. E., and Ryu, S. (2019). Default mode network connectivity is associated with long-term clinical outcome in patients with schizophrenia. NeuroImage Clinical 22:101805. doi: 10.1016/j.nicl.2019.101805

Lorenz, I., Müller, N., Schlee, W., Hartmann, T., and Weisz, N. (2009). Loss of alpha power is related to increased gamma synchronization-A marker of reduced inhibition in tinnitus? Neurosci. Lett. 453, 225-228. doi: 10.1016/j.neulet.2009. 02.028

Luan, Y., Wang, C., Jiao, Y., Tang, T., Zhang, J., and Teng, G.-J. (2019). Prefrontaltemporal pathway mediates the cross-modal and cognitive reorganization in sensorineural hearing loss with or without tinnitus: a multimodal MRI study. Front. Neurosci. 13:222. doi: 10.3389/fnins.2019.00222

Lv, H., Wang, Z., Tong, E., Williams, L. M., Zaharchuk, G., Zeineh, M., et al. (2018). Resting-state functional MRI: everything that nonexperts have always wanted to know. AJNR Am. J. Neuroradiol. 39, 1390-1399.

Lv, H., Zhao, P., Liu, Z., Wang, G., Zeng, R., Yan, F., et al. (2016). Frequencydependent neural activity in patients with unilateral vascular pulsatile tinnitus. Neural Plast. 2016:4918186.

Mantini, D., Perrucci, M. G., Del Gratta, C., Romani, G. L., and Corbetta, M. (2007). Electrophysiological signatures of resting state networks in the human brain. Proc. Natl. Acad. Sci. U.S.A. 104, 13170-13175. doi: 10.1073/pnas.0700668104

Maudoux, A., Lefebvre, P., Cabay, J. E., Demertzi, A., Vanhaudenhuyse, A., Laureys, S., et al. (2012). Connectivity graph analysis of the auditory resting state network in tinnitus. Brain Res. 1485, 10-21. doi: 10.1016/j.brainres.2012. 05.006

Maudoux, A., Lefebvre, P., Cabay, J.-E., Demertzi, A., Vanhaudenhuyse, A., Laureys, S., et al. (2017). Auditory resting-state network connectivity in tinnitus: a functional MRI study. PLoS One 7:e36222. doi: 10.1371/journal.pone.003 6222

Metherate, R., and Cruikshank, S. J. (1999). Thalamocortical inputs trigger a propagating envelope of gamma-band activity in auditory cortex in vitro. Exp. Brain Res. 126, 160-174. doi: 10.1007/s002210050726

Minami, S. B., Oishi, N., Watabe, T., Uno, K., and Ogawa, K. (2018). Auditory related resting state fMRI functional connectivity in tinnitus patients: tinnitus diagnosis performance. Otol. Neurotol. 39, 1-5. doi: 10.1097/mao. 0000000000001626

Nathan, W., Stephan, M., Marcus, M., Katalin, D., and Thomas, E. (2005). Tinnitus perception and distress is related to abnormal spontaneous brain activity as measured by magnetoencephalography. PLoS Med. 2:e153. doi: 10.1371/ journal.pmed.0020153
Ochi, R., Noda, Y., Tsuchimoto, S., Tarumi, R., Honda, S., Matsushita, K., et al. (2020). White matter microstructural organizations in patients with severe treatment-resistant schizophrenia: a diffusion tensor imaging study. Prog. Neuro Psychopharmacol. Biol. Psychiatry 100:109871. doi: 10.1016/j.pnpbp. 2020.109871

Olivito, G., Clausi, S., Laghi, F., Tedesco, A. M., Baiocco, R., Mastropasqua, C., et al. (2017). Resting-state functional connectivity changes between dentate nucleus and cortical social brain regions in autism spectrum disorders. Cerebellum 16, 283-292. doi: 10.1007/s12311-016-0795-8

Ovidiu, K., Roland, S., Richard, K., and Manfred, G. (2006). Course of hearing loss and occurrence of tinnitus. Hear. Res. 221, 59-64. doi: 10.1016/j.heares.2006. 07.007

Pattyn, T., Van Den Eede, F., Vanneste, S., Cassiers, L., Veltman, D., Van De Heyning, P., et al. (2016). Tinnitus and anxiety disorders: a review. Hear. Res. 333, 255-265. doi: 10.1016/j.heares.2015.08.014

Pegge, S. A., Steens, S. C., Kunst, H. P., and Meijer, F. J. (2017). Pulsatile tinnitus: differential diagnosis and radiological work-up. Curr. Radiol. Rep. 5:5.

Pinto, P. C. L., Marcelos, C. M., Mezzasalma, M. A., Osterne, F. J. V., De Melo Tavares De Lima, M. A., and Nardi, A. E. (2014). Tinnitus and its association with psychiatric disorders: systematic review. J. Laryngol. Otol. 128, 660-664. doi: $10.1017 / \mathrm{s} 0022215114001030$

Pulec, J. L. (1995). Cochlear nerve section for intractable tinnitus. Ear Nose Throat J. 74, 468-476. doi: 10.1177/014556139507400708

Rajmohan, V., and Mohandas, E. (2007). The limbic system. Indian J. Psychiatry $49,132-139$.

Rauschecker, J. P., Leaver, A. M., and Muhlau, M. (2010). Tuning out the noise: limbic-auditory interactions in tinnitus. Neuron 66, 819-826. doi: 10.1016/j. neuron.2010.04.032

Rauschecker, J. P., May, E. S., Maudoux, A., and Ploner, M. (2015). Frontostriatal gating of tinnitus and chronic pain. Trends Cogn. Sci. 19, 567-578. doi: 10.1016/ j.tics.2015.08.002

Rodrigo, A., Laurent, R., Laurence, D., Monique, D., Daniela, E.-K., Naïma, D., et al. (2018). A key role of the prefrontal cortex in the maintenance of chronic tinnitus: an fMRI study using a Stroop task. NeuroImage Clin. 17, 325-334. doi: 10.1016/j.nicl.2017.10.029

Sair, H. I., Yahyavi-Firouz-Abadi, N., Calhoun, V. D., Airan, R. D., Agarwal, S., Intrapiromkul, J., et al. (2016). Presurgical brain mapping of the language network in patients with brain tumors using resting-state fMRI: comparison with task fMRI. Hum. Brain Mapp. 37, 913-923. doi: 10.1002/hbm.23075

Savastano, M. (2008). Tinnitus with or without hearing loss: are its characteristics different? Eur. Arch. Oto Rhino Laryngol. 265, 1295-1300. doi: 10.1007/s00405008-0630-z

Schmidt, S. A., Akrofi, K., Carpenter-Thompson, J. R., and Husain, F. T. (2013). Default mode, dorsal attention and auditory resting state networks exhibit differential functional connectivity in tinnitus and hearing loss. PLoS One 8:e76488. doi: 10.1371/journal.pone.0076488

Schmidt, S. A., Carpenter-Thompson, J., and Husain, F. T. (2017). Connectivity of precuneus to the default mode and dorsal attention networks: a possible invariant marker of long-term tinnitus. Neuroimage Clin. 16, 196-204. doi: 10.1016/j.nicl.2017.07.015

Schmidt, S. A., Zimmerman, B., Bido Medina, R. O., Carpenter-Thompson, J. R., and Husain, F. T. (2018). Changes in gray and white matter in subgroups within the tinnitus population. Brain Res. 1679, 64-74. doi: 10.1016/j.brainres.2017. 11.012

Seydell-Greenwald, A., Leaver, A. M., Turesky, T. K., Morgan, S., Kim, H. J., and Rauschecker, J. P. (2012). Functional MRI evidence for a role of ventral prefrontal cortex in tinnitus. Brain Res. 1485, 22-39. doi: 10.1016/j.brainres. 2012.08.052

Seydell-Greenwald, A., Raven, E. P., Leaver, A. M., Turesky, T. K., and Rauschecker, J. P. (2014). Diffusion imaging of auditory and auditory-limbic connectivity in tinnitus: preliminary evidence and methodological challenges. Neural Plast. 2014:145943.

Shore, S. E., and Wu, C. (2019). Mechanisms of noise-induced tinnitus: insights from cellular studies. Neuron 103, 8-20. doi: 10.1016/j.neuron.2019.05.008

Shulman, G. L., Fiez, J. A., Corbetta, M., Buckner, R. L., Miezin, F. M., Raichle, M. E., et al. (1997). Common blood flow changes across visual tasks: II. Decreases in cerebral cortex. J. Cogn. Neurosci. 9, 648-663. doi: 10.1162/jocn. 1997.9.5.648 
Sven, V., Kathleen, J., Jan, O., and Dirk, D. R. (2018). Influencing connectivity and cross-frequency coupling by real-time source localized neurofeedback of the posterior cingulate cortex reduces tinnitus related distress. Neurobiol. Stress 8, 211-224. doi: 10.1016/j.ynstr.2016.11.003

Sven, V., Paul, V. D. H., and Dirk, D. R. (2011). The neural network of phantom sound changes over time: a comparison between recent-onset and chronic tinnitus patients. Eur. J. Neurosci. 34, 718-731. doi: 10.1111/j.1460-9568.2011. 07793.x

Trevis, K. J., Mclachlan, N. M., and Wilson, S. J. (2016). Cognitive mechanisms in chronic tinnitus: psychological markers of a failure to switch attention. Front. Psychol. 7:1262. doi: 10.3389/fpsyg.2016.01262

Trevis, K. J., Tailby, C., Grayden, D. B., Mclachlan, N. M., Jackson, G. D., and Wilson, S. J. (2017). Identification of a neurocognitive mechanism underpinning awareness of chronic tinnitus. Sci. Rep. 7, 1-9.

van der Loo, E., Congedo, M., Vanneste, S., Van De Heyning, P., and De Ridder, D. (2011). Insular lateralization in tinnitus distress. Auton. Neurosci. Basic Clin. 165, 191-194. doi: 10.1016/j.autneu.2011.06.007

Vanneste, S., and De Ridder, D. (2012). The auditory and non-auditory brain areas involved in tinnitus. An emergent property of multiple parallel overlapping subnetworks. Front. Syst. Neurosci. 6:31. doi: 10.3389/fnsys.2012. 00031

Vanneste, S., and De Ridder, D. (2016). Deafferentation-based pathophysiological differences in phantom sound: tinnitus with and without hearing loss. NeuroImage 129, 80-94. doi: 10.1016/j.neuroimage.2015.12.002

Vanneste, S., To, W. T., and De Ridder, D. (2019). Tinnitus and neuropathic pain share a common neural substrate in the form of specific brain connectivity and microstate profiles. Prog. Neuro Psychopharmacol. Biol. Psychiatry 88, 388-400. doi: 10.1016/j.pnpbp.2018.08.015

Vossel, S., Geng, J. J., and Fink, G. R. (2014). Dorsal and ventral attention systems: distinct neural circuits but collaborative roles. Neuroscientist 20, 150-159. doi: $10.1177 / 1073858413494269$

Weissman, J. L., and Hirsch, B. E. (2000). Imaging of tinnitus: a review. Radiology $216,342-349$.
Xu, J. J., Cui, J. L., Feng, Y., Yong, W., Chen, H. Y., Chen, Y. C., et al. (2019). Chronic tinnitus exhibits bidirectional functional dysconnectivity in frontostriatal circuit. Front. Neurosci. 13:1299. doi: 10.3389/fnins.2019.01299

Yeo, C. J. J., Hutton, G. J., and Fung, S. H. (2018). Advanced neuroimaging in Balo's concentric sclerosis: MRI, MRS, DTI, and ASL perfusion imaging over 1 year. Radiol. Case Rep. 13, 1030-1035. doi: 10.1016/j.radcr.2018.04.010

Zheng, W., Peng, Z., Pengfei, Z., Jing, L., Heyu, D., Hongxia, Y., et al. (2019). Long-term reactions to pulsatile tinnitus are marked by weakened short-range functional connectivity within a brain network in the right temporal lobe. J. Magn. Reson. Imaging 49, 1629-1637. doi: 10.1002/jmri.26545

Zhou, G. P., Shi, X. Y., Wei, H. L., Qu, L. J., Yu, Y. S., Zhou, Q. Q., et al. (2019). Disrupted intraregional brain activity and functional connectivity in unilateral acute tinnitus patients with hearing loss. Front. Neurosci. 13:1010. doi: 10.3389/fnins.2019.01010

Zimmerman, B., Finnegan, M., Paul, S., Schmidt, S., Tai, Y., Roth, K., et al. (2019). Functional brain changes during mindfulness-based cognitive therapy associated with tinnitus severity. Front. Neurosci. 13:747. doi: 10.3389/fnins. 2019.00747

Zimmerman, B. J., Abraham, I., Schmidt, S. A., Baryshnikov, Y., and Husain, F. T. (2019). Dissociating tinnitus patients from healthy controls using resting-state cyclicity analysis and clustering. Netw. Neurosci. 3, 67-89. doi: 10.1162/netn_ a_00053

Conflict of Interest: The authors declare that the research was conducted in the absence of any commercial or financial relationships that could be construed as a potential conflict of interest.

Copyright (C) $2021 \mathrm{Hu}, \mathrm{Cui}, \mathrm{Xu}$, Yin, Wu and Qi. This is an open-access article distributed under the terms of the Creative Commons Attribution License (CC BY). The use, distribution or reproduction in other forums is permitted, provided the original author(s) and the copyright owner(s) are credited and that the original publication in this journal is cited, in accordance with accepted academic practice. No use, distribution or reproduction is permitted which does not comply with these terms. 